\title{
Quantum Monte Carlo Simulation of the High-Pressure Molecular-Atomic Crossover in Fluid Hydrogen
}

\author{
Kris T. Delaney, ${ }^{1}$ Carlo Pierleoni, ${ }^{2}$ and D. M. Ceperley ${ }^{1}$ \\ ${ }^{1}$ Department of Physics, University of Illinois at Urbana-Champaign, Urbana, IL 61801, USA \\ ${ }^{2}$ INFM-SOFT and Dipartimento di Fisica, Università dell'Aquila, I-67010 L'Aquila, Italy
}

(Dated: June 3, 2018)

\begin{abstract}
A first-order liquid-liquid phase transition in high-pressure hydrogen between molecular and atomic fluid phases has been predicted in computer simulations using ab initio molecular dynamics approaches. However, experiments indicate that molecular dissociation may occur through a continuous crossover rather than a first-order transition. Here we study the nature of molecular dissociation in fluid hydrogen using an alternative simulation technique in which electronic correlation is computed within quantum Monte Carlo, the so-called Coupled Electron Ion Monte Carlo (CEIMC) method. We find no evidence for a first-order liquid-liquid phase transition.

PACS numbers: 61.20.Ja, 62.50.+p, 64.70.Ja, 77.84.Bw
\end{abstract}

The behavior of hydrogen under a range of thermodynamic conditions is an important problem in theoretical physics, as well as in planetary science and highpressure physics. Hydrogen exhibits a rich variety of properties including several molecular and non-molecular phases, 1] a fluid metal-insulator transition, 2] and possible fluid 3] and superconducting [4] phases at low temperature. There exists much uncertainty about the equilibrium properties of high-pressure phases, including the structure and relative stabilities of a number of solid phases, [5, [6] the specific shape of the molecular-solid melting curve and the reason for the density maximum, 3 and the conditions of molecular dissociation in the fluid.

Shock-wave experiments [2] have hinted that the metalization of hydrogen in the liquid state occurs in a continuous manner at temperatures above 3000K. However, recent computer simulations [3, 7] using Car-Parrinello molecular dynamics (CPMD) with density functional theory within the local density approximation (DFTLDA) and generalized gradient approximation (DFTGGA) predict that the molecular dissociation in the fluid happens through a first-order phase transition, with discontinuous metalization, at temperatures lower than $3000 \mathrm{~K}$. These results could be reconciled by having the critical point for the transition at $T<3000 \mathrm{~K}$. In addition, other simulations of hydrogen fluid phases, which do not directly address the nature of the molecular dissociation, have been reported [8].

In this Letter, we present the results of a study of this transition employing a new quantum Monte Carlo (QMC) method, coupled electron-ion Monte Carlo (CEIMC). [9, 10] The first QMC approaches for studying high pressure hydrogen were limited to studying solid phases at $T=0 \mathrm{~K}$ : variational Monte Carlo (VMC) and diffusion quantum Monte Carlo (DMC) 11, 12]. Finite-temperature restricted path integral Monte Carlo (RPIMC) was used to investigate the possibility of a hydrogen liquid-liquid phase transition [13], finding a continuous molecular dissociation at $T \simeq 10000 \mathrm{~K}$ in agree- ment with experiments. 2] However, RPIMC becomes inefficient and inaccurate at lower temperatures.

The CEIMC approach, in contrast with earlier QMC methods, involves simultaneous evolution of separate but coupled Monte Carlo simulations for the electronic and ionic subsystems, which may then be treated on different levels of approximation. A large gain in computational efficiency can be made for lower temperatures by employing the Born-Oppenheimer approximation (BOA) and requiring that the electronic subsystem remain in its ground state $(T=0 \mathrm{~K})$. The ionic system is treated at finite temperature either as a set of classical particles, or quantum mechanically using the imaginary-time path integral formalism. This decoupling is good provided the thermal excitation of electrons is a small effect, a fact that holds if $T \ll T_{F}$, where $T_{F}$ is the Fermi temperature. For the densities of interest, those at which molecules dissociate in the fluid, $T_{F}>200,000 \mathrm{~K}$. The quality of the BOA applied to high-pressure hydrogen fluid has been tested, and found to be good, by comparing CEIMC to accurate RPIMC calculations, which contain no such approximation, at temperatures of 5,000 and $10,000 \mathrm{~K}$. 14] We therefore anticipate that the BOA will be even more accurate at lower temperatures.

For classical nuclei in the canonical ensemble, one can generate the configuration-space probability distribution by proposing a move from configuration $S[15]$ to configuration $S^{\prime}$ with uniform probability and using the Metropolis acceptance probability

$$
A=\min \left[1, \exp \left(-\beta \Delta\left(S, S^{\prime}\right)\right)\right],
$$

where $\beta$ is the inverse temperature, $\left(k_{B} T\right)^{-1}$, and $\Delta\left(S, S^{\prime}\right)$ the difference in BO energies of nuclear configurations $S$ and $S^{\prime}$, computed with a $T=0 \mathrm{~K}$ QMC method. The bias resulting from the statistical noise of $\Delta$ is reduced with correlated sampling $[9,10]$, and eliminated by using the penalty method [16].

For electronic sampling, we use either VMC or reptation quantum Monte Carlo (RQMC). RQMC [17] is 
a projector method that does not suffer from the mixedestimator bias of DMC and allows efficient calculations of energy differences. To handle electron antisymmetry, we use the fixed-phase method [18] which allows the modulus of the many-electron wavefunction to be fully optimized while the phase is held fixed. This gives an estimate of the ground state energy much lower than that of the variational estimate (by typically $2500 \mathrm{~K} /$ atom) but above that of the exact ground state energy. Analysis of related electron systems suggest that the error of the fixedphase energy will be between $10 \%$ and $30 \%$ of the VMC error, [20] i. e., between $250 \mathrm{~K} /$ atom and $750 \mathrm{~K} /$ atom. We expect relative errors, between different proton configurations, to be even less. 21] We also employ twist-averaged boundary conditions (TABC) 19 in the electronic calculation to greatly reduce finite-size effects, a problem that has caused substantial errors in earlier simulations of fluid hydrogen. 22] Well-converged energies and correlation functions are achieved using, depending on the density, between 108 and 500 twist angles.

In the CEIMC approach, one is free to choose any trial wavefunction for the electronic ground-state. An important consideration is the computer time required per step, since the wavefunction must be calculated for many nuclear configurations. When studying dense liquid hydrogen, we require a general wavefunction that is equally accurate for both molecular and non-molecular configurations. Therefore, we employ a fast band-structure calculation with an effective one-electron potential designed to produce accurate single-particle orbitals for a SlaterJastrow type wavefunction. Within the fixed-phase approach, it is only the orbitals that affect the systematic bias. One such set of orbitals would be those computed within Kohn-Sham theory. However, full self-consistency for each proton displacement would be too expensive for generating a large number of nuclear configurationspace samples. Consequently, we use a bare electronproton potential for the effective single-particle potential. Further screening and correlation effects are introduced with a Slater-Jastrow wavefunction; the Jastrow factor is from the RPA pseudopotential, including the one-body (electron-proton) term. 12 Such an approach is surprisingly good, as demonstrated by Figure 1 which shows VMC and RQMC total energies for five different frozen nuclear configurations. This trial function is comparable in quality to one using Kohn-Sham LDA orbitals in the Slater determinant for all configurations and densities tested, while being faster to generate. It is also more transferable than both localized molecular orbitals [9] (which cannot be used for non-molecular systems) and analytic backflow 23] (which is highly accurate only for high-density metallic systems).

We investigate the atomic-molecular transition in liquid hydrogen at fixed density $\left(1.35 \leq r_{s} \leq 1.55\right.$ where $\left.4 \pi r_{s}^{3} / 3=1 / n_{e}\right)$ and temperature $(T=2000 \mathrm{~K}$ and $1500 \mathrm{~K})$. The pressure, estimated using the virial the-

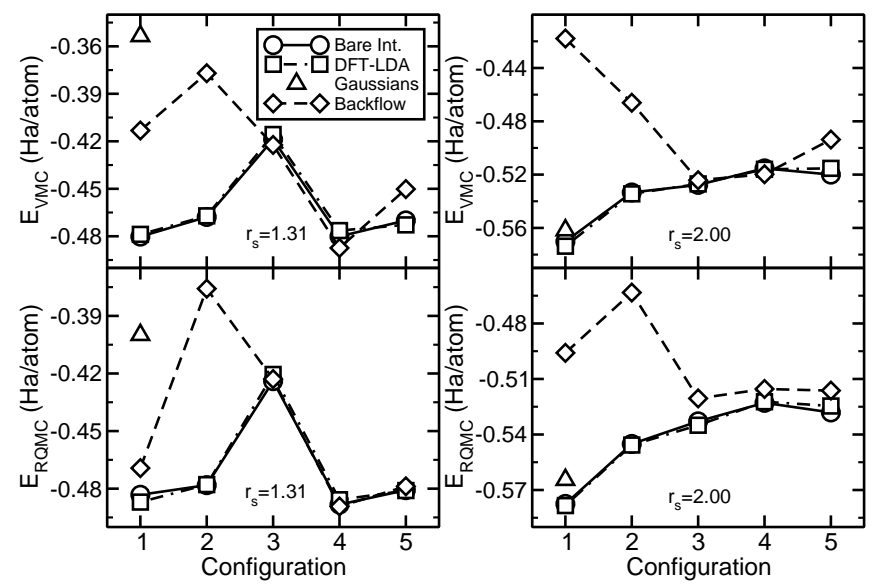

FIG. 1: VMC and RQMC total energies for five different crystal configurations at two different densities (left graphs $V=$ 9.42a.u./atom or $r_{s}=1.31$; right graphs $V=33.51 \mathrm{a} . \mathrm{u}$./atom or $\left.r_{s}=2.0\right)$ using a number of different Slater-Jastrow wavefunctions. Configuration 1 is molecular, $2-5$ are nonmolecular. Owing to the variational principle, a lower energy implies a better wavefunction. DFT-LDA and bare electronproton bands (see text) provide the best and most transferable orbitals for the Slater determinant. Gaussian for configuration 1 refers to localized molecular orbitals.

orem, an approach that is accurate with RQMC due to the lack of a mixed-estimator bias, lies between $135 \mathrm{GPa}$ and $290 \mathrm{GPa}$ for this range of densities. Using a classical Monte Carlo simulation with an empirical potential, 10] the system is prepared either in a purely molecular or a purely atomic fluid initial configuration. During the subsequent simulation the system evolves to its equilibrium state, subject to overcoming any free-energy barriers during the simulation. A typical simulation has 14,000 ionic moves with a step size of $0.006-0.016 \mathrm{Bohr}$. We collect statistics along the sequence of ionic states, particularly the proton-proton correlation functions which give insight into the state of the liquid through a distinctive peak at $r_{p p} \sim 1.4 \mathrm{Bohr}$ when molecules are present. Figure 2 shows a set of proton-proton correlation functions for simulations at several densities prepared with either a molecular or atomic fluid as the initial state.

For a quantitative analysis, a method for estimating the average number of molecules from the paircorrelation function at each phase point is required. A rough estimate would involve integrating the paircorrelation function up to the first minimum, but such an approach does not take into account the "baseline" caused by nearby molecules and unbound atoms; see Fig. 2. To remove the baseline, we fit each of the paircorrelation functions to the functional form:

$$
g(r)=\lambda g_{\mathrm{m}}(r ;\{\alpha\})+(1-\lambda) g_{\mathrm{at}}(r ;\{\gamma\}),
$$

where $\{\alpha, \gamma\}$ are fitting parameters and $g_{\mathrm{m}}$ is a Gaussian centered on $1.3-1.5 \mathrm{Bohr}$. $g_{\text {at }}$ is a fit to the next-nearest 


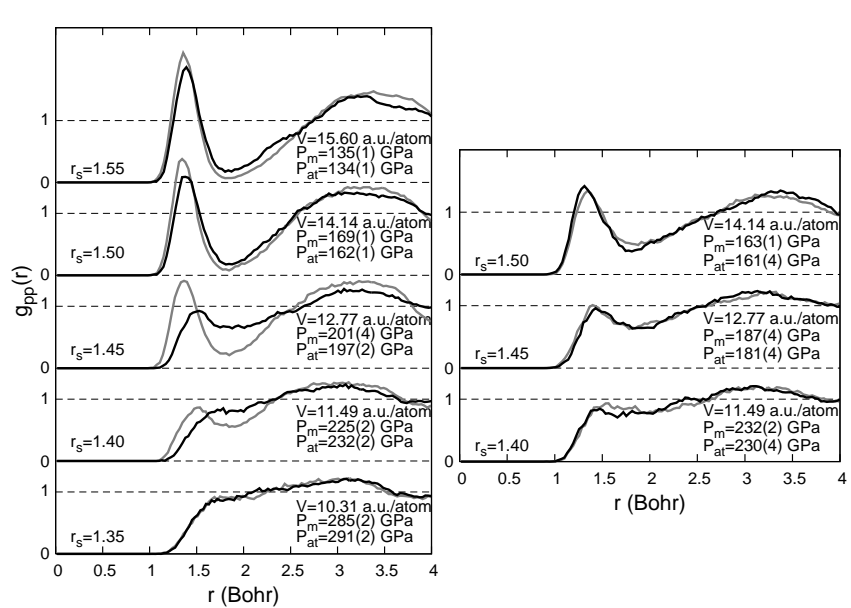

FIG. 2: Proton-proton correlation functions for CEIMC simulations in the canonical ensemble with 32 atoms at $T=$ 2000K. The BO energies are computed with VMC (left pane) or RQMC (right pane) and the wavefunction is a SlaterJastrow type with bare electron-proton bands (see text). Grey lines are simulations initially prepared with a molecular fluid and black with an atomic fluid. Hysteresis is evident. $P_{\text {at }}$ and $P_{\mathrm{m}}$ are the computed pressures for simulations prepared with an atomic and a molecular fluid respectively. All correlation functions are plotted to the same scale.

neighbor distribution. We find that the quality of the fit is insensitive to the choice of $g_{\text {at }}$; the molecular fraction varies by no more than $3 \%$ for different choices. The molecular order parameter, $\lambda$, the fraction of protons that are bound into a molecule, is plotted against pressure in Fig. 3 for $T=2000 \mathrm{~K}$.

We find that the CEIMC simulations using VMC energy differences (left pane Fig. 2, and Fig. 33) yield an irreversible transition with hysteresis. This implies a freeenergy barrier that is difficult to overcome during the simulations, hence a metastable state is obtained. The large width of the hysteresis loop is indicative of a transition that is weakly first-order. We therefore expect that $T=2000 \mathrm{~K}$ is close to the VMC critical point.

In contrast, the much more accurate RQMC simulations (right pane Fig. 2, and Fig. 3) yield a reversible and continuous molecular dissociation upon increasing pressure. Both molecular and atomic states are mechanically unstable in the range of densities simulated, and the system quickly evolves, with no discernible free-energy barrier, to a stable part-molecular, part-atomic state. This behavior implies the lack of an underlying first-order transition, a behavior which would not change as the thermodynamic limit is approached. The likely reasons for the change in character of the crossover is that the BO energy surface of the assumed Slater-Jastrow trial function is inaccurate under conditions at which molecules are short-lived transient entities. As discussed above, these deficiencies are corrected by the imaginary-time projection of RQMC, if they are due to errors in the modulus

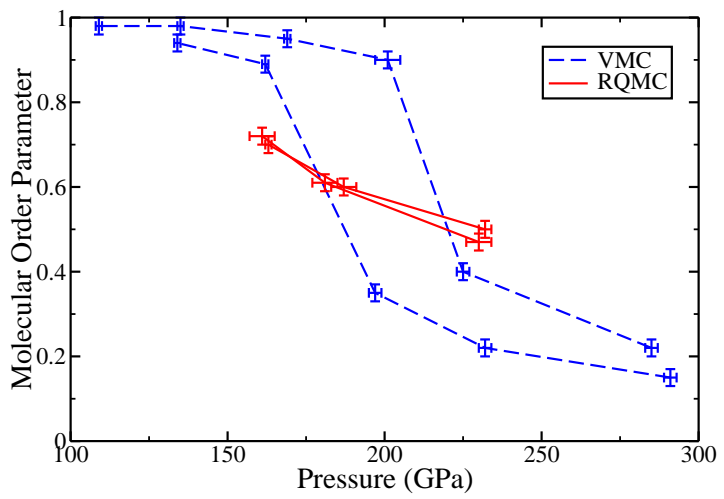

FIG. 3: Molecular order parameter ( $\lambda$ in Eq. 2) evaluated for simulation results from Fig. 2. Two curves are obtained for each method from different initial conditions (see text). VMC (dashed lines) simulations indicate an irreversible, weakly first-order phase transition for molecular dissociation in the fluid with increasing/decreasing pressure. This picture is compatible with conclusions from CPMD DFT-LDA. Accurate RQMC (solid lines) simulations find a reversible crossover.

of the trial wavefunction and not its phase.

We have assessed the finite-size errors in the cell volume by repeating a selection of simulations with 54 atoms. The proton-proton correlation functions in Figure 4 show the comparison between 32- and 54-atom simulations for VMC and RQMC. For VMC finite-size errors are clearly large, consistent with the presence of a free-energy barrier, while for RQMC the errors are small, indicative of a continuous crossover between molecular and atomic states.

We have tested the nature of molecular dissociation at a lower temperature: $T=1500 \mathrm{~K}$. Figure 5 shows pair-correlation functions. Hysteresis is again evident for VMC simulations, although over a shorter pressurerange, indicating a larger free-energy barrier. Again, RQMC simulations do not show a first-order transition, mirroring the behavior at $T=2000 \mathrm{~K}$.

In conclusion, this study demonstrates that the CEIMC approach with a fast and accurate trial wavefunction can be used to determine the nature of the pressure-driven molecular to atomic crossover in hydrogen for temperatures on the order of $1000 \mathrm{~K}$. We find that the improvements in interatomic interactions obtained when using RQMC to simulate the electronic subsystem lead to no first-order phase transition, a result of fundamentally different nature to that predicted when using VMC. Our most accurate simulations, those employing RQMC, provide no evidence for a first-order atomic-molecular transition in the liquid phase at either $T=1500 \mathrm{~K}$ or $T=2000 \mathrm{~K}$, showing instead a continuous crossover; this is in contrast to the first-order transition found with CPMD. The remaining uncontrolled approximations in our simulations are the finite-size error (demonstrated to be small for RQMC), the fixed-phase 

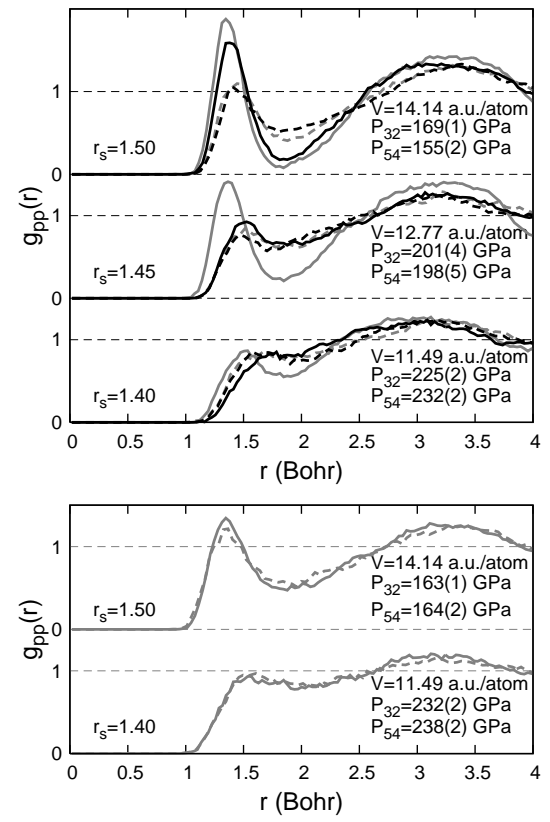

FIG. 4: Demonstration of finite-size errors in VMC paircorrelation functions (upper pane). Solid lines are 32-atom simulations, dashed are 54 atoms. Grey lines are simulations started from a molecular fluid and black are from an atomic fluid. Quoted pressures are for simulations started from a molecular fluid. RQMC (lower pane) simulations show small finite-size errors.

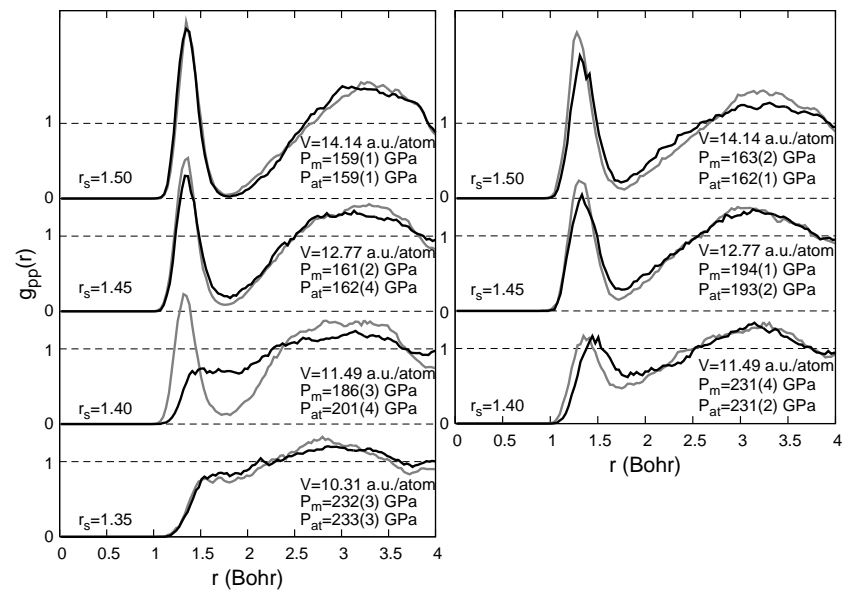

FIG. 5: Proton-proton correlation functions for simulations at $T=1500 \mathrm{~K}$. Black lines are simulations prepared with an atomic fluid, grey are prepared with a molecular fluid. The simulations demonstrate an irreversible transition with hysteresis for VMC. Left pane: VMC; Right pane: RQMC.

approximation in RQMC, the adiabatic approximation for decoupling nuclear and electronic time-scales (tested in ref. 14] ) and the use of classical statistics for simulating nuclei. Path integral calculations are in progress for studying the effect of nuclear quantum statistics on dense liquid hydrogen. We anticipate that nuclear quan- tum effects will destabilize molecules at a lower pressure leaving the crossover character unchanged.

This material is based upon work supported by the NSF awards DMR 04-04853 and DMR 03-25939 ITR, and by MIUR-COFIN2003. Calculations were performed at the NCSA at the University of Illinois at UrbanaChampaign and at CINECA (Italy). We thank S. Chiesa and D. Quigley for fruitful discussions.

[1] H.-K. Mao and R.J. Hemley, Rev. Mod. Phys. 66, 671 (1994).

[2] S.T. Weir et al., Phys. Rev. Lett. 76, 1860 (1996).

[3] S.A. Bonev et al., Nature 431, 669 (2004).

[4] E. Babaev et al., Nature 431, 666 (2004).

[5] V. Natoli, R.M. Martin and D. Ceperley, Phys. Rev. Lett. 74, 1601 (1995); K.A. Johnson and N.W. Ashcroft, Nature 403, 632 (2000).

[6] S. Biermann et al., Solid State Comm. 108, 337 (1998); Jorge Kohanoff et al., Phys. Rev. Lett. 78, 2783 (1997); Jorge Kohanoff et al., Phys. Rev. Lett. 83, 4097 (1999).

[7] S. Scandolo, PNAS 100, 3051 (2003).

[8] Ali Alavi et al., Phys. Rev. Lett. 73, 2599 (1994); Jorge Kohanoff et al., Phys. Rev. E 54, 768 (1996); Stefan Nagel et al., Phys. Rev. E 57, 5572 (1998).

[9] M. Dewing and D.M. Ceperley, in Recent Advances in quantum Monte Carlo Methods, II, edited by S. Rothstein (World Scientific, Singapore, 2003).

[10] D.M. Ceperley, M. Dewing and C. Pierleoni, Bridging Time Scales, edited by P. Niebala et al., Lecture Notes in Physics Vol. 605 (Springer-Verlag, Berlin, 2003); C. Pierleoni and D.M. Ceperley, to appear in "Lecture Notes in Physics" (2006), arXiv:physics/0510254

[11] D.M. Ceperley and B.J. Alder, Phys. Rev. B 36, 2092 (1987).

[12] D.M. Ceperley and B.J. Alder, Physica 108B, 875 (1981).

[13] B. Militzer and D.M. Ceperley, Phys. Rev. E 63, 066404 (2001). W.R. Magro et al., Phys. Rev. Lett. 76, 1240 (1996).

[14] C. Pierleoni, D.M. Ceperley and M. Holzmann, Phys. Rev. Lett. 93, 146402 (2004).

[15] $S$ is the collection of all nuclear coordinates in the simulation, $S=\left\{\mathbf{R}_{i}\right\}$, where $\mathbf{R}_{i}$ is the position of the $i^{\text {th }}$ nucleus.

[16] D.M. Ceperley and M. Dewing, J. Chem. Phys. 110, 9812 (1999).

[17] S. Baroni and S. Moroni, Phys. Rev. Lett. 82, 4745 (1999).

[18] G. Ortiz, D.M. Ceperley and R.M. Martin, Phys. Rev. Lett. 71, 2777 (1993).

[19] C. Lin, F.H. Zong and D.M. Ceperley, Phys. Rev. E 64, 016702 (2001).

[20] Y. Kwon, D.M. Ceperley and R.M. Martin, Phys. Rev. B 58, 6800 (1998).

[21] C. Pierleoni and D.M. Ceperley, Chem. Phys. Chem. 6, 1872 (2005).

[22] D. Hohl et al., Phys. Rev. Lett. 71, 541 (1993).

[23] M. Holzmann et al., Phys. Rev. E 68, 046707 (2003). 